\title{
Erratum to: Biological pathways and genetic variables involved in pain
}

\author{
Qiuling Shi · Charles S. Cleeland · Pål Klepstad • \\ Christine Miaskowski • Nancy L. Pedersen • \\ The GeneQOL Consortium
}

Published online: 18 November 2010

(C) Springer Science+Business Media B.V. 2010

\section{Erratum to: Qual Life Res (2010) 19:1407-1417 \\ DOI 10.1007/s11136-010-9738-x}

In the original publication The GeneQOL Consortium was missing as the last author, and so was the corresponding list of participants and their affiliations. They are included in this erratum.

\section{GENEQOL Consortium participants per March 2009}

Amy P. Abernethy, Duke Cancer Care Research Program, Duke University Medical Center, Durham, NC, US; Frank

The online version of the original article can be found under doi:10.1007/s11136-010-9738-x.

\section{Q. Shi $(\bowtie) \cdot$ C. S. Cleeland}

Department of Symptom Research, The University of Texas MD Anderson Cancer Center, 1515 Holcombe Blvd, Unit 1450,

Houston, TX 77030, USA

e-mail: qshi@mdanderson.org

C. S. Cleeland

e-mail: ccleeland@mdanderson.org

P. Klepstad

Department of Anesthesiology, University Hospital of

Trondheim, Olav Kyrres gt. 17, 7006 Trondheim, Norway

e-mail: Pal.Klepstad@ntnu.no

\section{Miaskowski}

Department of Physiological Nursing, UCSF School of Nursing, Box 0610, San Francisco, CA 94143-0610, USA

e-mail: chris.miaskowski@nursing.ucsf.edu

\section{N. L. Pedersen}

Department of Medical Epidemiology and Biostatistics, Karolinska Institutet, PO Box 281, 17177 Stockholm, Sweden e-mail: Nancy.Pedersen@ki.se
Baas, Laboratory of Neurogenetics, Academic Medical Center, University of Amsterdam, Amsterdam, The Netherlands; Andrea M. Barsevick, Nursing Research and Education, Fox Chase Cancer Center, Philadelphia, PA, US; Meike Bartels, Department of Biological Psychology, VU University, Amsterdam, the Netherlands; Dorret I. Boomsma, Department of Biological Psychology, VU University, Amsterdam, the Netherlands; Cynthia Chauhan, Cancer Advocay, Wichita, KS, US; Charles S. Cleeland, Department of Symptom Research, The University of Texas M. D. Anderson Cancer Center, Houston, TX, US; Amylou C. Dueck, Section of Biostatistics, Mayo Clinic, Scottsdale, AZ, US; Marlene H. Frost, Women's Cancer Program, Mayo Clinic, Rochester, MN, US; Per Hall, Department of Medical Epidemiology and Biostatistics, Karolinska Institute, Stockholm, Sweden; Michele Y. Halyard, Department of Radiation Oncology, Mayo Clinic, Scottsdale, AZ, US; Pål Klepstad, Department of Intensive Care Medicine, St Olavs University Hospital, Norwegian University of Technology and Science, Trondheim, Norway; Nicholas G. Martin, Queensland Institute of Medical Research, Brisbane, Australia; Christine Miaskowski, School of Nursing, University of California, San Francisco, CA, US; Miriam Mosing, Queensland Institute of Medical Research, Brisbane, Australia; Benjamin Movsas, Department of Radiation Oncology, Henry Ford Health System, Detroit, MI, US; Cornelis J. F. Van Noorden, Department of Cell Biology and Histology, Academic Medical Center, University of Amsterdam, Amsterdam, The Netherlands; Donald L. Patrick, Department of Health Services, University of Washington, Seattle, WA, US; Nancy L. Pedersen, Department of Medical Epidemiology and Biostatistics, Karolinska; Institute, Stockholm, Sweden; Mary E. Ropka, Cancer Prevention and Control Program, Fox 
Chase Cancer Center, Cheltenham, PA, US; Qiuling Shi, Department of Symptom Research, The University of Texas M. D. Anderson Cancer Center, Houston, TX, US; Gen Shinozaki, Department of Psychiatry and Psychology, Mayo Clinic, Rochester, MN, US; Jasvinder A. Singh, Minneapolis Veterans Affairs Medical Center and University of Minnesota, Minneapolis, MN and Mayo Clinic College of Medicine, Rochester, MN, US; Jeff A. Sloan, Department of Health Sciences Research, Mayo Clinic, Rochester, MN, US; Mirjam A. G. Sprangers,
Department of Medical Psychology, Academic Medical Center, University of Amsterdam, Amsterdam, The Netherlands; Ruut Veenhoven, Faculty of Social Sciences, Erasmus University Rotterdam, Rotterdam, The Netherlands; Ping Yang, Department of Genetic Epidemiology, Mayo Clinic, Rochester, MN, US; Ailko H. Zwinderman, Department of Clinical Epidemiology and Biostatistics, Academic Medical Center, University of Amsterdam, Amsterdam, The Netherlands. 